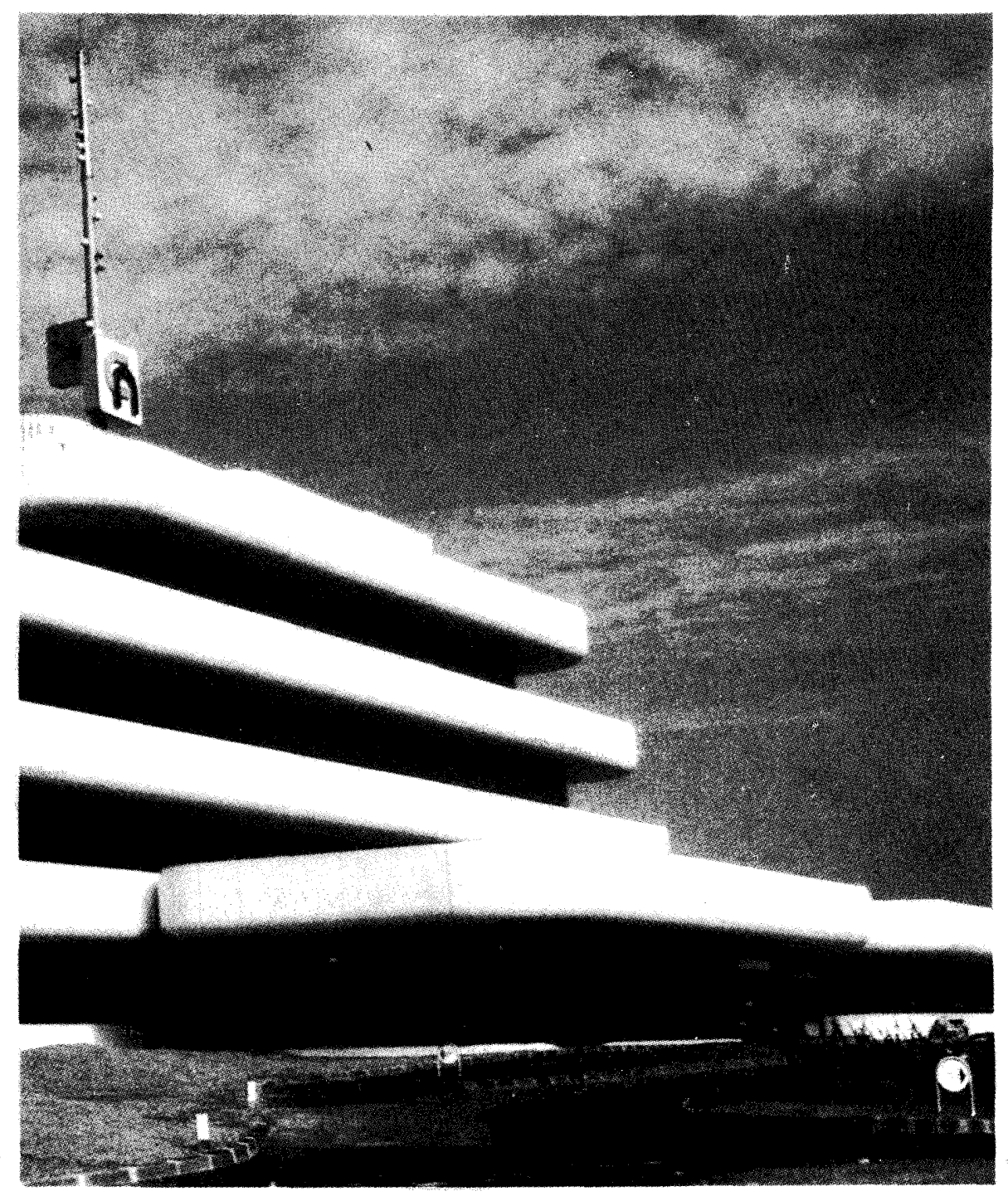

\title{
plásticos armados o con carga mineral en Construcción y Obras Públicas
}

G. Vie, Ingeniero de Minas

$882-3$

\section{sinopsis}

En este artículo se hace un análisis de los plásticos armados más utilizados en la Construcción y Obras Públicas, que son aquellos en los que el material de refuerzo es la fibra de vidrio. El elemento principal es una resina sintética, sólida o líquida, termoplástica o termoendurecida.

Entre los usos de estos productos destacan: las placas translúcidas de vidrio-poliéster para paneles solares; la resina aminoplasta armada de vidrio para el sostenimiento de hastiales y bóvedas en las excavaciones subterráneas; el cloruro de polivinilo plastificado con incrustaciones de áridos duros para firmes antideslizantes; el revestimiento «gel-coab» para polivinilo plastificado con incrustaciones de aridos duros para firmes antideslizantes; el revestimiento "gel-coab» para
protección contra el frío de los hormigones; los paneles de poliestireno y poliuretano, tipo «sandwich», utilizados en la industria del frío; etcétera.

Además de los plásticos armados se estudia el vidrio de recuperación obtenido, en gran parte, de los residuos urbanos aprovechables. 


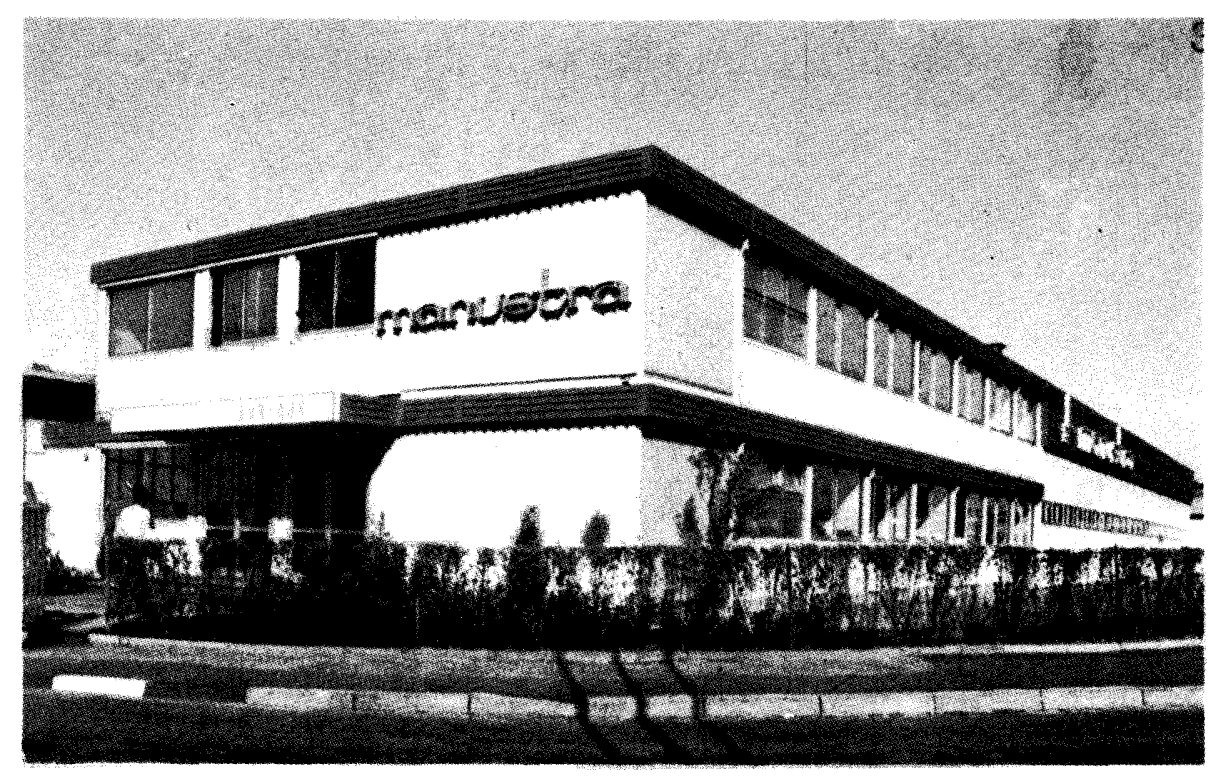

Las aplicaciones de las resinas sintéticas bajo la denominación de «plásticos» son cada vez más numerosas en Edificación y Obras Públicas, sobre todo en forma de «materiales reforzados».

Los refuerzos con fibras de vidrio proporcionan a los plásticos características nuevas de resistencia.

Las fibras de vidrio se obtienen por el paso, a través de una hilera, de vidrio fundido de una composición particular, por ejemplo, un borosilicato.

Las fibras de vidrio más usuales son las formadas por filamentos continuos, aunque para algunas aplicaciones particulares se utilizan también fibras de filamentos discontinuos.

En el momento del fibrado se depositan en la superficie de los filamentos de vidrio unos productos lubrificantes con objeto de conseguir un buen enlace químico con las resinas sintéticas que esas fibras están destinadas a reforzar.

Se encuentran en el mercado en forma de ovillos o bobinas de hilos cortados, en fibras de hilos triturados, en fieltros de hilos cortados y bobinas de hilos rizados, y también en bobinas de cintas torsionadas o no.

A partir de estas últimas formas se pueden obtener tejidos mixtos.

En la fabricación del material compuesto, la resina sintética, sólida o líquida, termoendurecida o termoplástica, juega el papel de elemento matriz.

Se pueden agregar a la resina líquida cargas minerales pulverulentas para obtener mejores características en el material final, consiguiéndose a menudo que baje el precio de coste del plástico armado.

Las resinas termoendurecidas, a las que se hace referencia en este artículo, son sobre todo las poliéster y, en menor grado, las epoxi, fenólicas, siliconas, melaminas.

En el curso de su puesta en obra sufren un cambio de estado irreversible, pasando del estado líquido mediante un tratamiento adecuado al estado sólido permanente.

Este cambio, en el caso de las resinas poliéster, viene dado por una copolimerización de poliésteres no saturados con un monómero íntimamente mezclado con ellos.

Esta polimerización que provoca el endurecimiento se efectúa en presencia de un catalizador activado, bien mediante un acelerador químico o bien por aporte de energía (calor, campo natural de alta frecuencia, etcétera). 
En la fabricación de los materiales de poliéster armado hay tres fases:

a) Impregnación por la resina líquida del refuerzo fibroso y eliminación del aire incorporado.

b) Formación del material compuesto en las dimensiones que se desee obtener.

c) Endurecimiento del compuesto por polimerización de la resina.

El precio de coste del producto baja si se hace una fabricación continua o automatizando las sucesivas operaciones.

El porcentaje de material de refuerzo que interviene en la fabricación varía entre el 25 y el $75 \%$ según la naturaleza y el destino final de las piezas compuestas.

Cuando el procentaje es alto, las tensiones de rotura a flexión y a tracción pueden alcanzar valores elevados.

En Alcalá de Henares (España) hay una fábrica de fibras de vidrio para plásticos armados.

La gran diversidad de usos en el campo de la construcción (edificación) condiciona la presentación y el aspecto.

Los productos pueden presentarse en forma plana, perfilados, en piezas moldeadas y volúmenes huecos, en elementos de gran talla (monobloques), ligeros, translúcidos u opacos, pintados en la superficie o coloreados en la masa.

No son susceptibles a la corrosión, ni a los ataques del agua, ni a la mayoría de los agentes químicos. Son también buenos aislantes térmicos y dan unos buenos resultados mecánicos.

Ningún arquitecto ignora las posibilidades de los plásticos en trabajos de cubierta, techos, solados, paneles, tabiques y puertas, ventanas, aparatos sanitarios, piscinas, señalización, etcétera.

La experiencia, en el caso de paneles solares, nos demuestra que se prefieren las placas translúcidas de vidrio-poliéster a cualquier otra.

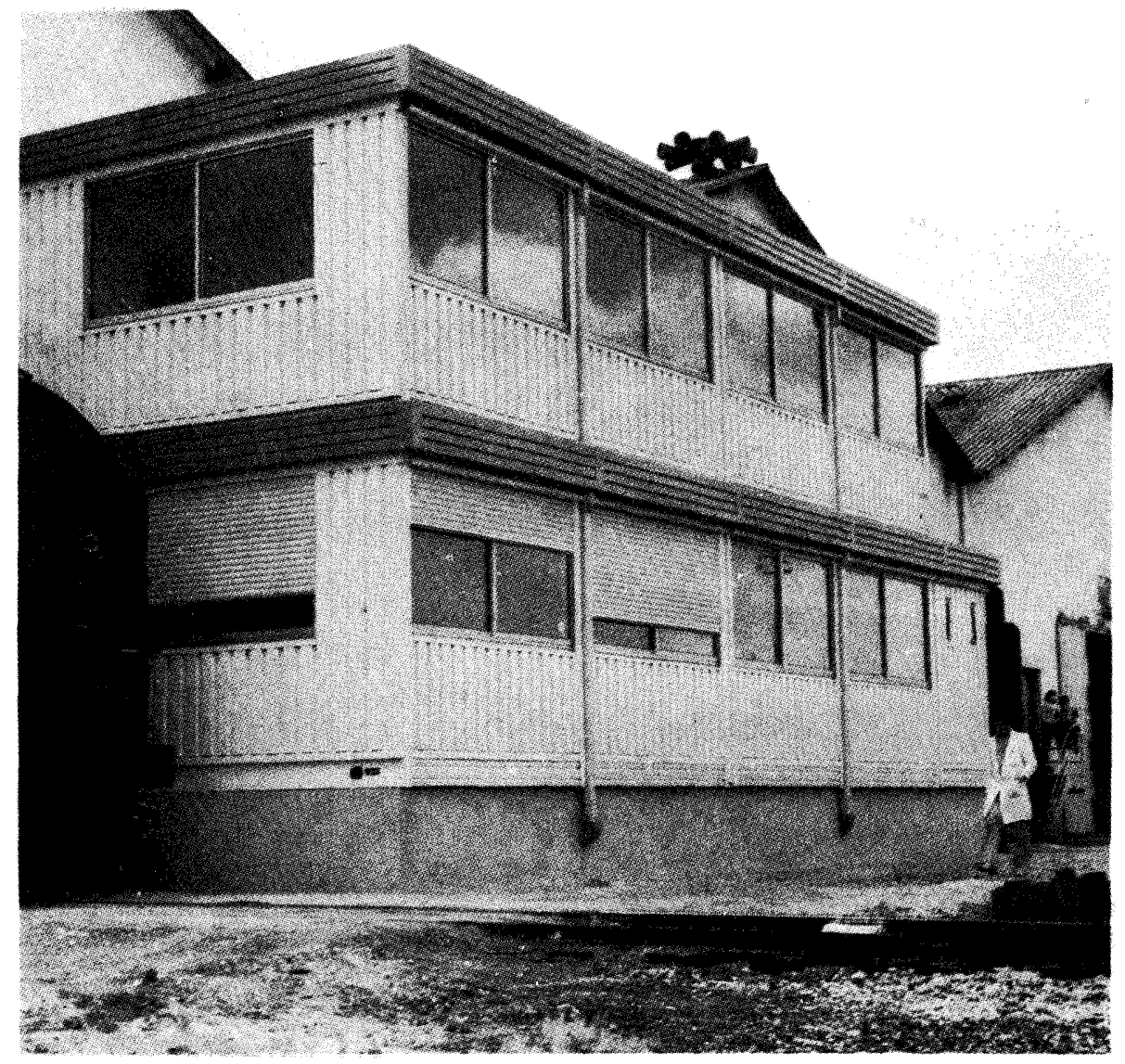


En efecto, de los estudios comparativos entre el plástico armado y el metacrilato de metilo, utilizados ambos en las células multiunión para concentración estática, los primeros han demostrado su superioridad.

Podrá abrirse de esta manera un vasto mercado de captación de energía solar en los países donde el soleamiento se manifiesta a lo largo de todo el año.

El arquitecto, al elegir la mejor disposición de un edificio, deberá tener en cuenta esta importante fuente de radiación.

Hace ya muchos años que el progreso de la tecnología nos ha llevado al uso de la técnica de proyección del hormigón para, en colaboración con fibras de acero de refuerzo, mejorar el comportamiento de terrenos en las minas, túneles de carretera y otras galerías en Obras Públicas.

Este tipo de sostenimiento es ventajoso, puesto que adquiere rápidamente una resistencia mecánica elevada. Su empleo, sin embargo, no ha sido tan amplio como se esperaba debido a que existen un cierto número de problemas prácticos de puesta en obra.

El hormigón se puede reemplazar, como en otras muchas circunstancias, por una resina sintética reforzada de una manera similar.

Otra técnica puesta a punto por una Sociedad Francesa, en colaboración con las Minas de Hierro de Lorraine, consiste en la pulverización de resina aminoplasta armada de vidrio.

Esta Sociedad tiene una alta especialización desde hace muchos años en la técnica de sostenimiento de hastiales y bóveda de una excavación subterránea por medio de bulones de anclaje en la resina.

Este nuevo método juega un papel muy importante en las aplicaciones siguientes:

- Protección de los terrenos en vía de descomposición bajo la acción del aire y de la humedad en las minas de hierro y en las explotaciones de bauxita.

- Sustitución de la malla metálica que se coloca asociada al bulonado para evitar el «descascarillado» de los terrenos. El experimento ha tenido un resultado favorable en algunas explotaciones hulleras, como Lorraine y Dauphine; en algunas explotaciones subterráneas de materias primas minerales, en galerías para aprovechamientos hidroeléctricos, etcétera.

En Lorraine, ese procedimiento se aplicó a la consolidación de los anillos de las galerías trazadas en pleno carbón, en la realización de muros de contención, contra el fuego de las minas, etcétera.

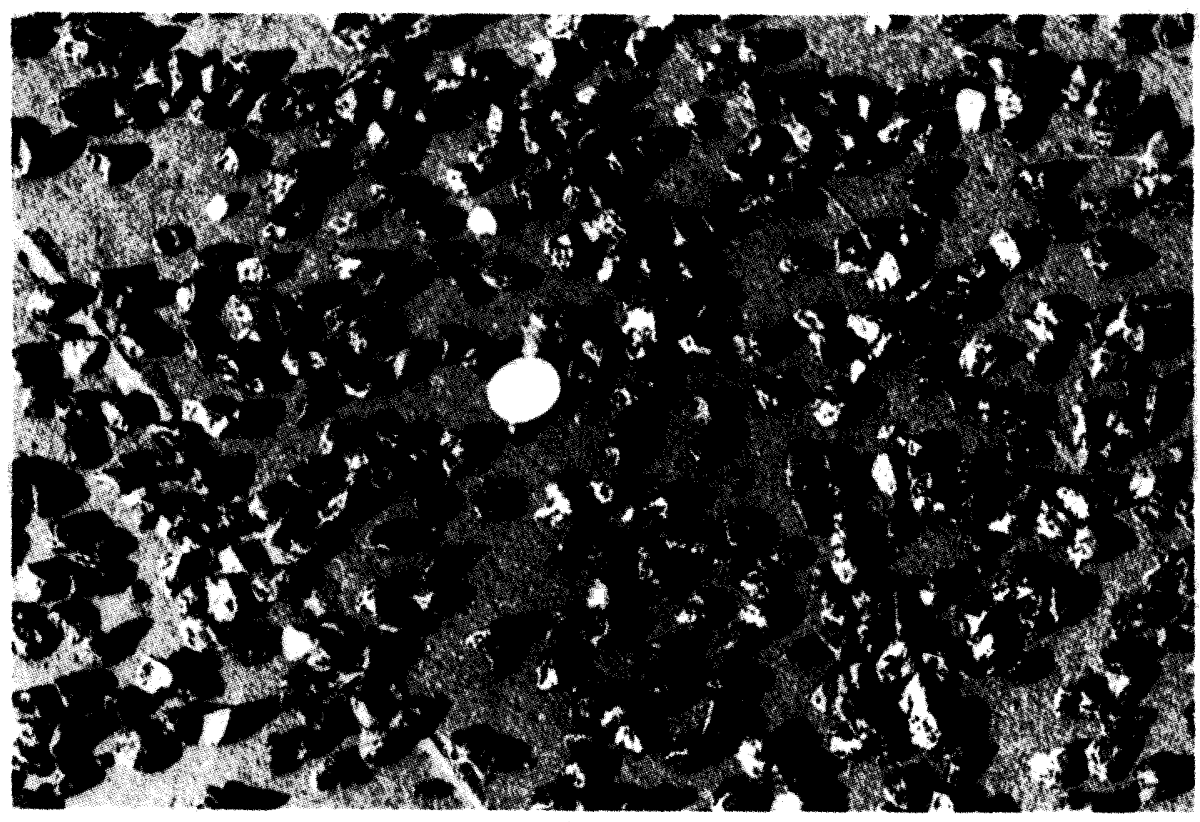

Gravilla semi-incrustada hasta un $\varnothing$ de $16 \mathrm{~mm}$. (Fotografía tomada antes del paso de un rodillo de cilindro liso.) 
Los materiales utilizados - resinas y fibras de vidrio- son ininflamables y no tóxicos en caso de incendio. Las calidades mecánicas de un revestimiento de este tipo son muy elevadas, y su espesor está en la relación de 1 a 10, si se compara con los revestimientos obtenidos por proyección de hormigón.

El precio de coste es también muy inferior.

Otra aplicación de los materiales plásticos, hasta hace poco bastante impensable, es la construcción de firmes antideslizantes.

Inspirándose en el revestimiento de las calzadas mediante hormigón bituminoso con incrustaciones de gravillas cúbicas de rocas duras, poco sensibles al pulimento por desgaste, el Laboratorio de Puentes y Calzadas de Francia ha puesto a punto un nuevo tipo de revestimiento prefabricado, destinado a aquellas zonas de las carreteras donde la resistencia al deslizamiento es primordial, como es el caso de los márgenes para paradas de urgencias, las proximidades de la señal "Stop», los semáforos, los puestos de peaje, etcétera.

Está constituido por un soporte colocado en caliente de cloruro de polivinilo PVC plastificado, en el cual están incompletamente empotrados áridos muy duros: rocas volcánicas o eruptivas, ofita, basaltos e incluso cuarcitas y pórfidos.

Tramos de ensayo instalados desde hace muchos años permanecen prácticamente intactos.

Variando las características de estos revestimientos, a través de la granulometría y la densidad de los elementos rocosos, el ruido provocado por la rodadura de los neumáticos varía en frecuencia, lo que produce un efecto sonoro que advierte al conductor de un coche su llegada a una zona peligrosa.

La adherencia o, más exactamente, el agarre de los neumáticos a una superficie de rodadura con esta rugosidad es tan grande, que ensayos voluntarios de deslizamiento lo único que han conseguido es quemar el caucho por recalentamiento.

La experiencia de los aglomerados bituminosos con incrustaciones de gravillas cúbicas ha servido para ese nuevo tipo de revestimiento de carreteras.

Es razonable recurrir, para un aglomerado soporte a base de resina sintética, a las mismas curvas granulométricas generalmente aceptadas para los citados aglomerados bituminosos con adición o no de fillers.

Los finos pueden ayudar a un buen engarce de los elementos rocosos.

La maquinaria para la ejecución de las diversas operaciones puede ser la misma que en el caso de los aglomerados bituminosos citados anteriormente, así como los porcentajes de áridos que conducen a niveles de adherencia satisfactoria, sin riesgo de rechazos. Este porcentaje de áridos varía entre 4 a $4,8 \mathrm{dm}^{3} / \mathrm{m}^{2}$, según la granulometría de los áridos.

Se utilizará, por ejemplo, rodillos lisos que aseguran la penetración de los áridos en el aalomerado soporte, es decir, el engarce.

No haremos aquí un estudio sobre los precios de coste respectivos, pues están bajo la influencia de muchos factores.

Una fórmula original de resina epoxi, en emulsión acuosa, permite sacar partido, con gran facilidad de empleo, de las propiedades de resistencia mecánica y química de las resinas epoxi en revestimiento delgado, alrededor de 0,2 mm, sin las restricciones de empleo en medio disolvente.

Ese producto de dos componentes se aplica sobre diferentes soportes, tales como hormigón, acero ordinario, aluminio. Se adhiere muy bien al acero galvanizado. Puede tener diversas tonalidades, aunque se usa sobre todo en tonos claros.

Se puede, por adición a este producto de polvo de corindón, realizar revestimientos de suelos antipolvos y antidesgaste en áreas hormigonadas, aparcamientos, depósitos, pistas, escalones, tribunas, piscinas, silos, garajes individuales, escaleras de servicio, balcones, etcétera.

Las características químicas de esta emulsión le permiten resistir a los detergentes y al agua de mar, y a la proyección de grasas, aceites e hidrocarburos. 
El abanico de usos puede ampliarse incluso a las galerías subterráneas, cuyos paramentos necesitan ser estancos. Los diferentes métodos adoptados hasta ahora: empleo de arcilla, proyección de yeso sobre una malla metálica, etcétera, necesitan el transporte de una importante cantidad de materiales.

Una espuma impregnada de plástico suprime este inconveniente gracias a la fabricación «in situ» de un revestimiento duro, impermeable y ligero. Una resina, pulverizada sobre una solución espumosa, endurece gracias al ácido contenido en ésta.

Los componentes se llevan a pie de obra en bidones. Dos bombas de aire comprimido envían los dos componentes a una cámara de mezclado, de donde se lanzan con la ayuda de una manguera sobre los paramentos. Para obtener $1 \mathrm{~m}^{3}$ de material se necesitan 15 I de solución espumosa y 30 I de una resina especial, la IGLONEIGE.

Algo parecido es el revestimiento "gel-coat», creado por los Laboratorios de Cementos Lafarge para protección de hormigones a los que da un aspecto de superficie lisa, brillante, mate o en relieve. Se usa en decoración, en paneles aislantes acústicos y en barreras de seguridad.

El frío es susceptible, en clima nórdico, de provocar microfisuras en los elementos de hormigón, con lo que después del deshielo la corrosión ataca las armaduras.

La aplicación del «gel-coat» se efectúa sobre los materiales durante el moldeado de prefabricación. Una vez que el molde está colocado se encera, antes de recibir una aplicación de "gel-coat» poliéster.

Después de la polimerización se aplica sobre esta capa una cola especial que estará en contacto con el cemento fresco.

La mayoría de las formas de las piezas son realizables, ya que el desmoldeo es facilitado por la película de cera.

En caso de accidente de la pieza, una vez acabada, la capa superficial se repara según las técnicas usuales de las resinas poliéster.

La capa de «gel-coat» puede soportar, sin deterioro alguno, un ciclo de hielos y deshielos. Es totalmente impermeable al agua.

Ensayos a tracción de las piezas ponen de manifiesto que la rotura se produce en el hormigón y no en las zonas encoladas.

Una protección eficaz contra la tendencia al envejecimiento de algunos plásticos se obtiene mediante la aplicación de una capa de polifluoruro de vinilideno, de algunas decenas de micrones de espesor.

Se usa principalmente en la protección de los termoplásticos: PVC, poliestireno, policarbonatos. Por ser el mismo polifluoruro un termoplástico, es de una puesta en obra fácil. Es resistente a los rayos ultravioleta y a la oxidación del oxígeno y del ozono. Es impermeable a los tensioactivos, hidrocarburos y disolventes.

Para los elementos más o menos importantes, destinados a la Construcción y a la Edificación, es posible producir estratificados de gran espesor por impregnación sucesiva de muchas capas de tejido de vidrio con una resina poliéster.

El espesor, 12 a $15 \mathrm{~mm}$, e incluso $25 \mathrm{~mm}$, se obtiene mediante un porcentaje bastante grande de fibras de vidrio y de la presencia eventual de cargas. Se puede, por ejemplo, incorporar hasta un $30 \%$ de aluminio, como carga sin afectar demasiado a la velocidad de polimerización y a las cualidades del estratificado.

La polimerización se simplifica y acelera por exposición bajo una lámpara de rayos ultravioleta. En menos de seis minutos, la resina está ya gelificada, y en diez minutos, el endurecimiento es total.

Esta duración puede ser aún menor si además de las lámparas de ultravioleta se usan lámparas de infrarrojos que aportan calor. Si agregamos menos del 0,50 \% de peróxido se produce una aceleración del endurecimiento. 
Aunque no se obtienen tan buenas cualidades mecánicas como con los tejidos reforzados con fibra de vidrio, las resinas poliamidas se pueden reforzar con microesferas de vidrio. En contrapartida, en el caso de piezas moldeadas, la retracción es isotrópica y las piezas no acusan deformación.

La adición de cargas minerales en partículas esféricas y no fibrosas, hasta un porcentaje del $40 \%$, mejora las características de rigidez y de resistencia a la tracción, y acelera la polimerización.

Se construyen con plásticos, cada vez más, canalizadores de sección mediana o grande.

La industria petrolífera, en su lucha contra la corrosión, se interesa por los plásticos armados en la industria química, ya utilizados desde hace veinte años.

Tanto en piezas de caldería en depósitos como en canalizaciones, se utilizan resinas epoxi superiores o resinas poliéster, alcanzándose buenas cualidades tanto desde el punto de vista de la resistencia mecánica como de la fluencia y de la resistencia a la corrosión.

Se utilizan resinas Epikote asociadas a un agente endurecedor, el diaminodifenolmetano, en la relación 100/27.

La armadura de refuerzo es una banda de fibras de vidrio, con un peso de $580 \mathrm{~g} / \mathrm{m}^{2}$, y de enrollamiento helicoidal.

La relación de fibras de vidrio/resina debe ser al menos igual a uno.

La densidad de la resina armada es del orden de $1.800 \mathrm{~kg} / \mathrm{m}^{3}$.

Los manguitos y las canalizaciones de una tubería se realizan con capas estratificadas de vidrio-resina.

Las capas interna y externa están constituidas por un $90 \%$ de resina, con lo que se asegura un buen comportamiento químico.

Consta de una membrana superficial, normalmente de vidrio, o de fibras orgánicas (PVC, acrílicas, etcétera).

La capa interna es a la vez una barrera anticorrosiva y una película de impermeabilidad.

La capa externa es una protección frente al medio ambiente.

La resistencia mecánica de los tubos (matriz de resina termoendurecida armada de fibras de vidrio) está asegurada por una pared reforzada.

Una de las aplicaciones más recientes de los plásticos es la de paneles aislantes de tipo «sandwich» para la industria del frío.

Muebles frigoríficos se prefabrican de poliuretano y poliestireno, inyectados entre dos chapas de acero.

El módulo básico sinve tanto para construir un depósito de $40.000 \mathrm{~m}^{3}$-como el realizado recientemente en Arabia Saudita - como para el simple mueble de conservación que tienen la mayoría de los comerciantes de artículos perecederos de consumo.

La unión entre módulos básicos es muy rápida; por ejemplo, el depósito de $40.000 \mathrm{~m}^{3}$ mencionado anteriormente lo montaron dos especialistas y un equipo de peones sin ninguna calificación.

Se puede citar, por último, aunque esté fuera de la materia principal que se acaba de tratar, el empleo de vidrio de recuperación obtenido en gran parte de los residuos urbanos aprovechables. Para poder llegar a un empleo cada vez mayor es necesaria la ayuda de los ciudadanos, que deben responder a las llamadas, en este sentido, de los Ayuntamientos.

El vidrio de recuperación, del cual cientos de toneladas van a las descargas de desperdicios cada día, se puede triturar finamente mezclado con un $2 \%$ de caliza. El polvo así obtenido, transformado en bolitas de diámetros variables, se somete en seguida al secado y al endurecimiento, pasando posteriormente al horno rotativo para realizar su expansión. 


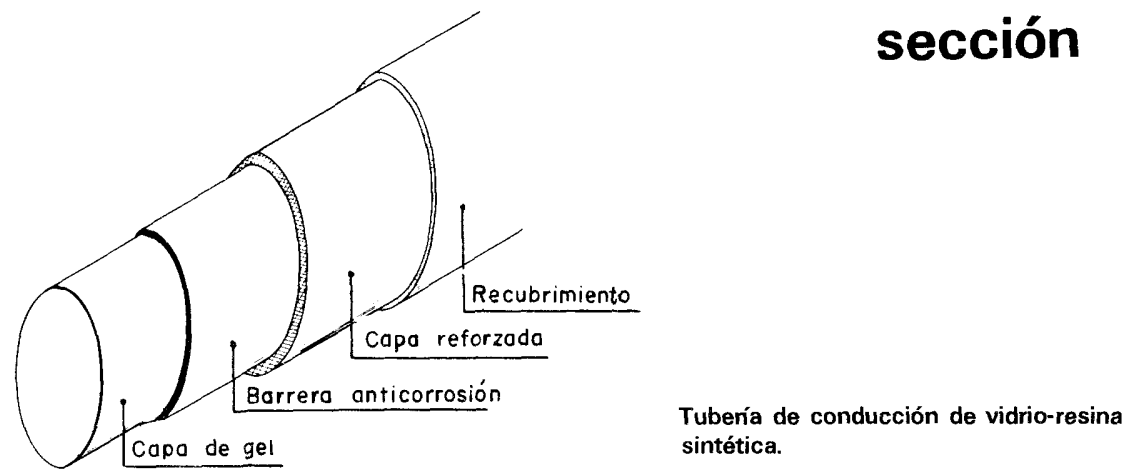

Se obtiene así un granulado de vidrio multicelular, con poros incomunicados con el exterior, de forma esférica y cuyos diámetros están comprendidos entre 3-8, 8-16 y 16-25 mm.

El enfriamiento controlado de esos granulados artificiales les confiere las características deseadas. Pueden servir para la preparación de hormigones celulares o semicelulares mediante un simple amasado en hormigonera corriente. En edificación se pueden usar también, como aislante térmico, en el yeso, para la construcción de tabiques.

Su incorporación a las resinas sintéticas es igualmente posible, bien mezclándolas in situ con aquéllas durante su vertido entre encofrados, bien para confeccionar elementos manufacturados (prefabricados).

Los elementos así realizados se caracterizan por su ligereza, resistencia a la humedad, a los agentes químicos y biológicos, excelente comportamiento al fuego, estabilidad dimensional y capacidad de aislamiento acústico.

La expansión del vidrio se realiza en las proximidades de los $800^{\circ} \mathrm{C}$, y los gases calientes son, a su vez, utilizados para las operaciones de sintetizado, secado y enfriamiento progresivo del producto.

Este ha sido el proceso de fabricación llevado a cabo en la Fábrica de ANICHE, en el Norte de Francia, a partir del cual se han obtenido apreciables economías de materias primas y de energía, lo que no deja de tener importancia en una época de crisis como la actual.

\section{résumé}

PLASTIQUES ARMES OU A CHARGE MINERALE EN CONSTRUCTION ET TRAVAUX PUBLICS

G. Vié, ingénieur des mines

Dans cet article, l'auteur fait une analyse des plastiques armés les plus utilisès en construction et en travaux publics, qui sont ceux dont le matériau de renfort est la fibre de verre. L'élément principal est une résine synthétique, solide ou liquide, thermoplastique ou thermodurcissable.

Parmi les applications de ces produits se distinguent les plaques translucides polyesterverre pour les panneaux solaires, la résine aminoplaste armée de verre qui, par scellement, sert à la technique du boulonnage des parements et de la calotte, en excavations souterraines, le chlorure de polyvinyle plastifié, dans lequel sont incomplètement enrobés des cailloux très durs, pour les revêtements antidérapants, la couche de "gel-coat» pour la protection des bétons contre le froid, les panneaux de polystyrène et polyuréthane, type «sandwich», pour l'industrie du froid, etc.

Enfin, en dehors du sujet principal, les plastiques armés, l'auteur attire l'attention sur l'utilisation du verre de récupération, collecté, en grande partie, dans les ordures ménagères.

\section{summary}

REINFORCED OR MINERAL FILLED PLASTICS IN CONSTRUCTION AND CIVIL ENGINEERING

G. Vié, mining engineer

This article analyses the most commonly used reinforced plastics in Construction and Civil Engineering works, where the reinforcing material is glass fiber. The basic material is a solid or liquid, thermoplastic or thermosetting synthetic resin.

The applications of these materials include: glasspolyester traslucid panel for solar panels: glass-reinforced aminoplastic resin used in gallery excavation to support vaults and checks; plastified vinyl-chloride with hard ag gregate embedded used for non-split roadbeds; gel-coating to protect concrete against cold weather; polyestyrene and polyurethane sandwich panels used in freezing industry, etc.

Besides reinforced plastics, attention is given to recycled glass obtained to a large extent from home refuse.

\section{zusammenfassung}

BEWEHRTE ODER MINERALVERSTARKTE KUNSTSTOFFE IM BAUWESEN UND FUR OFFENTLICHE BAUTEN

G. Vié, Bergingenieur

In diesem Artikel werden die am meisten im Bauwesen und für öffentliche Bauten eingesetzten bewehrten Kunststoffe untersucht. Es sind diejenigen, bei welchen als Verstärkungsmaterial Glasfasern zum Einsatz gelangen Das Hauptelement ist ein festes oder flüssiges, thermoplastiches oder thermogehärtetes Kunstharz.

Unter den Anwendungsgebieten dieser Produkte sind zu erwähnen: die durchsichtigen Polyesterglastafeln für Sonnenplatten; das glasverstärkte Aminoplastharz für die Abstützung von Streckenstössen und Gewölben bei unterirdischen Aushebungen; plastifiziertes Polyvinylchlorid mit harten Zuschlagstoffen für nichtgleitende Strassendecken; die (GelCoat»)-Verkleidung für Betonkälteschutz; die in der Kälteindustrie zum Einsatz gelangenden Polystvrol und Polyurethantafeln, «SandwichType»), usw.

Ausser den bewehrten Kunststoifen wird das zum grossen Teil aus vewertbarum Stadtmül zurückgewonnene Glas betrachtet. 